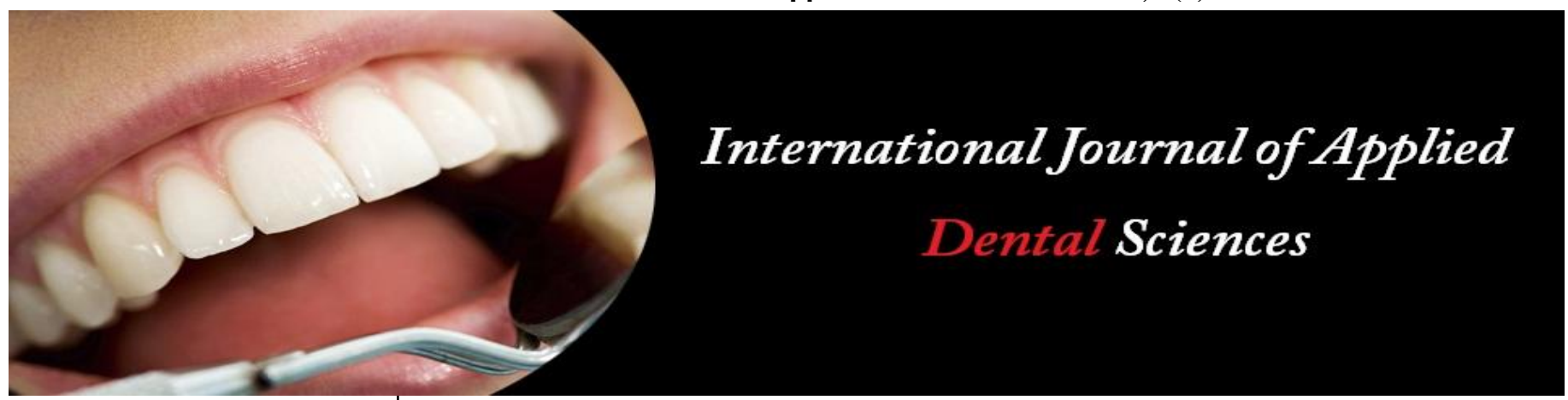

ISSN Print: 2394-7489

ISSN Online: 2394-7497

IJADS 2020; 6(4): 296-300

(C) 2020 IJADS

www.oraljournal.com

Received: 13-09-2020

Accepted: 15-10-2020

Dr. Mishan Manohar Jaiswal

M.D.S, Conservative Dentistry

and Endodontics, Senior

Resident, Department of

Dentistry, Nalanda Medical

College \& Hospital, Bihar, India

Dr. Neha Kumari

D.S, Pedodontics and Preventive

Dentistry, Dental Practitioner.

Consultant Pedodontist, Dental

Life Care, Param Parwati

market, 1st Floor, Vijay Nagar,

Bailey Road, Patna, Bihar, India

Dr. Sarita Singh

M.D.S, Prosthodontics and

Crown \& Bridge, Senior

Resident, Department of

Dentistry, Nalanda Medical

College \& Hospital, Patna,

Bihar, India
Corresponding Author: Dr. Sarita Singh M.D.S, Prosthodontics and Crown \& Bridge, Senior Resident, Department of Dentistry, Nalanda Medical College \& Hospital, Patna, Bihar, India
Original article

\section{Evaluation of microscopic flora and its antibiotic susceptibility from RCT failure cases: An in vivo study}

\author{
Dr. Mishan Manohar Jaiswal, Dr. Neha Kumari and Dr. Sarita Singh
}

DOI: $\underline{\text { https://doi.org/10.22271/oral.2020.v6.i4e.1080 }}$

\section{Abstract}

Background: The persistence of disease in periapical tissues is most commonly associated with difficulties or mishaps which occur during or after initial endodontic treatment.

Aims and Objective: To isolate and identify the organisms in root canal failed cases \& its current antibiotic susceptibility pattern.

Materials and Methods: study was conducted on 51 patients attending the OPD of dental Department Nalanda Medical College and Hospital, Patna. The results were analyzed using VITEK-2 automated analyser (Bio Merieux UK).

Results: A total of 18 types of bacteria were isolated and among them $17.3 \%$ cases reported E- faecalis and another $15.2 \%$ cases reported E-cloacae complex. Pseudomonas aeruginosa accounted for $8.7 \%$ while $6.5 \%$ constituted of E-faecium, Escherichia coli etc.

Conclusion: Enterococcus species was found in majority of cases indicates that it is the predominant bacteria responsible for endodontic failure.

Keywords: Micro-organisms, RCT, vitek-2

\section{Introduction}

It is evident that an infected root canal system is a unique niche for the selective species of microorganisms ${ }^{[1]}$. The influence of bacterial persistence in the root canals on treatment outcome is an important issue in endodontics because bacteria have been shown to play a major role in persistence or emergence of apical periodontitis lesions after root canal treatment [2]. The continuity of periapical diseases is mostly associated with strain or mishaps which occur during or after endodontic treatment. Insufficient aseptic control, poor access cavity design, missed canals, inadequate instrumentation and leaking temporary or permanent restorations are the main factors that may be critically important in post treatment disease ${ }^{[3]}$. Despite thorough mechanical instrumentation and disinfection of the root canal system, microbes have been recovered at subsequent treatment sessions and at the end of the treatment. Such residual organisms and the leakage of bacterial products are likely to play a role in treatment failures ${ }^{[4,5]}$. Recently growing significance has been placed on extraradicular infection as a cause of persisting apical periodontitis. The extraradicular occurrence of several anaerobic bacteria has been reported in post-treatment apical periodontitis lesions. A number of potentially endodontopathic bacteria have been found in asymptomatic periapical inflammatory lesions which were refractory to endodontic treatment. From active site of periodontal diseases anaerobic black-pigmented Bacteroides species and anaerobic grampositive rods and cocci have been isolated. Facultative Streptococcus species have also been isolated, but appear to play a minor role ${ }^{[6]}$. In the case of prolonged endodontic therapy, the antibiotic prescription can play an important role in efficient treatment. Recently, technological advances in bacterial isolation have made the dental profession aware that anaerobic microorganisms predominate in infected root canals.

\section{Aims and objectives}

1. To isolate and identify the organisms in root canal failed cases.

2. To know the predominant organisms causing root canal failure. 
3. To determine the current antibiotic susceptibility pattern of the isolates from failed endodontic treatment cases.

\section{Methodology}

The study was conducted on 51 patients attending the OPD of dental Department Nalanda Medical College and Hospital, Patna.

None of the patients had received any systemic antibiotic therapy in the preceding 3 months. All of the teeth had demonstrated radiographic signs of chronic apical periodontitis. All patients had completed root canal therapy more than 1 year previously. All the samples were taken with the sterile instrument.

A rubber dam was applied, and the operating field was disinfected using moller's protocol. The root filling was removed using Gates Glidden drills, protaper retreatment files, Hedstrom file and $\mathrm{K}$ files without the use of chemical solvent under irrigation with sterile saline. The root canal length was measured with electronic apex locator and a small sterile $\mathrm{K}$ file wherever possible without use of any irrigant. After that a $2 \mathrm{ml}$ disposable syringe with 21 gauge needle was taken. Sterile normal saline was drawn into canal.The solution inside the canal was absorbed onto 2 or 3 sterile paper points for 30 seconds.

For aerobic culture $50 \%$ of the samples were studied for identification of isolates by gram stains and culture growth on nutrient, blood and MacConkey agar. Colonies from nutrient agar was used for biochemical tests and antibiotic sensitivity. The remaining $50 \%$ of the samples was placed into the BacT/ALERT aerobic transport medium bottle and sent for culture and sensitivity by using VITEK-2 automated analyser. For anaerobic culture, sample was placed into BacT/ALERT Anaerobic transport medium bottle which was immediately sent to the microbiological laboratory. In the laboratory, the BacT/ALERT automated culture instrument was used for routine culture. The predominant isolate obtained after culture was evaluated for routine microbial identification and susceptibility testing using the VITEK-2 automated analyser (Bio Merieux, UK).

\section{Result}

46 cases showed growth of microorganisms out of 51 cases. In 5 cases there was no growth. The predominant isolate bacteria was analyzed in each case and total of 18 types of bacteria were isolated and among them $17.3 \%$ cases reported Enterococcus faecalis and another $15.2 \%$ cases reported Enterobacter cloacae complex. Pseudomonas aeruginosa accounted for $8.7 \%$ while $6.5 \%$ constituted of Enterococcus faecium, Escherichia coli, Klebsiella pneumonia, Staphy lococcus hominis. Acinobacter baumannii, Ralstonia pickettii, Staphylococcus aureus and streptococcus salivarius accounted for $4.3 \%$. Candida albicans, Citrobacter freundii, Morganella morganii species, Pantoea species, Pseudomonas putida, Staphylococcus epidermidis, Staphylococcus lentus accounted for $2.1 \%$ each.

In analyzing the prevalence of bacterial genera in failed root canal Enterococcus accounted for $23.9 \%$ of cases, Enterobacter and Staphylococcus $15.2 \%$ of the cases, Pseudomonas 10.8\%, Echerichia and Klebsiella 6.5\%, Acinetobacter, Ralstonia and Streptococcus 4.3\%, Candida, Citrobacter, Morganella and Pantoea $2.1 \%$ of the cases.
Further more in analyzing the bacteria present in failed root canal cases on gram staining, it was found that $55.5 \%$ cases were gram negative and $44.4 \%$ were gram positive.

Facultative anaerobic bacteria were more prevalent with $88.8 \%$ and obligate aerobes were constituted $11.1 \%$.

\section{Discussion}

In the present study, Enterococcus faecalis was the most commonly found microorganism. The common recovery of E.faecalis from canals where the treatment has failed suggests that it is an opportunistic pathogen whose persistence in the root canal presents a significant therapeutic problem ${ }^{[7]}$. In this study, they were isolated from $17.3 \%$ of the canals with bacterial growth.

This finding agrees with those reported by Molander et al. ${ }^{[8]}$ and Sundquist et al. ${ }^{[9]}$, who respectively found E.faecalis in 47 and $38 \%$ of previously root treated canals with positive culture. Peciuliene et al. ${ }^{[10,11]}$ reported (in two studies) a higher isolation frequency of this microorganism: 70 and $64 \%$. According to Hung-Chih Chien et al. 83 of $111(74.7 \%)$ teeth demonstrated a positive culture, in which viridans group streptococci were most abundant, with an isolation rate of $54.1 \%$ (60 of 111 specimen). The isolation rate of Enterococcus was $12.6 \%$ (13/111), while both F.nucleatum and Pre.intermedia were found in $8.1 \%$ (9/111) of isolates. Other microorganisms including Pep.micros and Pre. Endodontalis, were also identified and isolation rates for these 2 organisms were $7.2 \%(8 / 111)$ and $4.5 \%$ (5/111), respectively ${ }^{[12]}$.

Our result showed that all $8 \mathrm{E}$. faecalis strains were sensitive to linezolid, teicoplanin and vancomycin, 6 (75\%) were sensitive penicillin, chloramphenicol, ampicillin and amoxyclav, $5(62.5 \%)$ were sensitive to tetracycline, 3 $(37.5 \%)$ were sensitive clindamycin and erythromycin. All were resistant to gentamycin.

Susceptibility and antibiotic sensitivity of different bacteria were analyzed and linezolid, teicoplanin \& vancomycin were the most effective antimicrobial against gram positive bacteria and Amikacin, Gentamycin, Cefotaxime, Cefepime, Cefoperazone, Piperacillin/tazobactam, Imipenem and Meropenam were the most effective antimicrobial against the gram negative bacteria for root canal failure.

According to Hung Chih Chien aminopenicillins exhibited good activity against the viridans group of streptococci and preendodontalis, new tetracycline derivatives, minocycline and doxycycline expressed very pronounced antimicrobial activity, metronidazole had less effect on facultatives such as viridans group streptococci and Enterococcus, clindamycin was inhibitory against most of the anaerobic and facultative organisms, of all the cephalosporins tested, 30\% species except pre intermedia were resistant to cephalexin, furthermore, $15.4 \%$ of Enterococcus and F.nucleatum were resistant to cefixime, $40 \%$ of organisms were resistant to erythromycin. Amomg the tested antibiotics trovafloxacin, a third generation fluoroquinolone showed the highest activity against all 6 species ${ }^{[12]}$.

According to study conducted by Heena Sadiq et al. it was found that the bacteria isolated from the root canals were commonly resistant to ampicillin, Erythromycin was relatively unaffected according to the results and clindamycin had shown to be effective against the pathogens of root canal in the study ${ }^{[13]}$. 


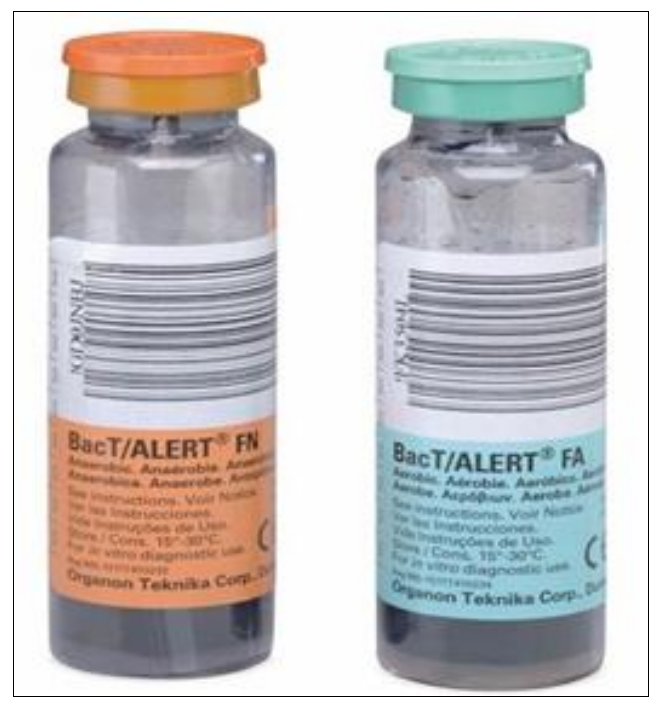

Fig 1: BacT/Alert Culture Bottle (Anaerobic \& Aerobic)

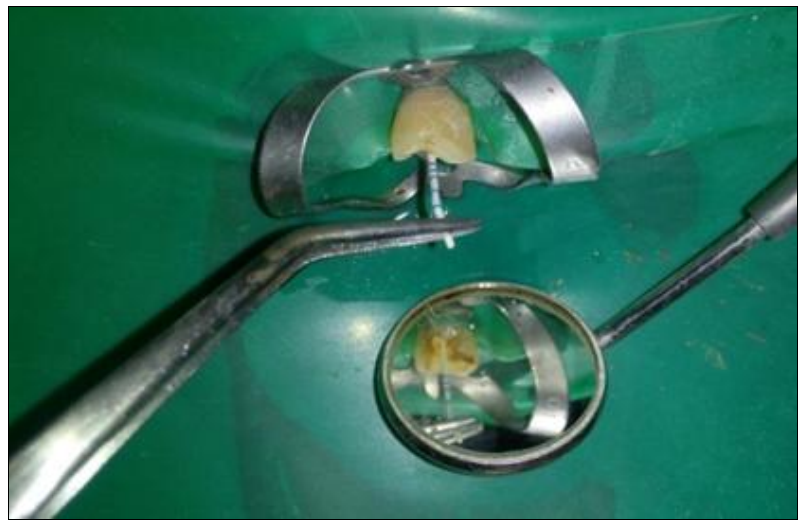

Fig 2: Sample Collection

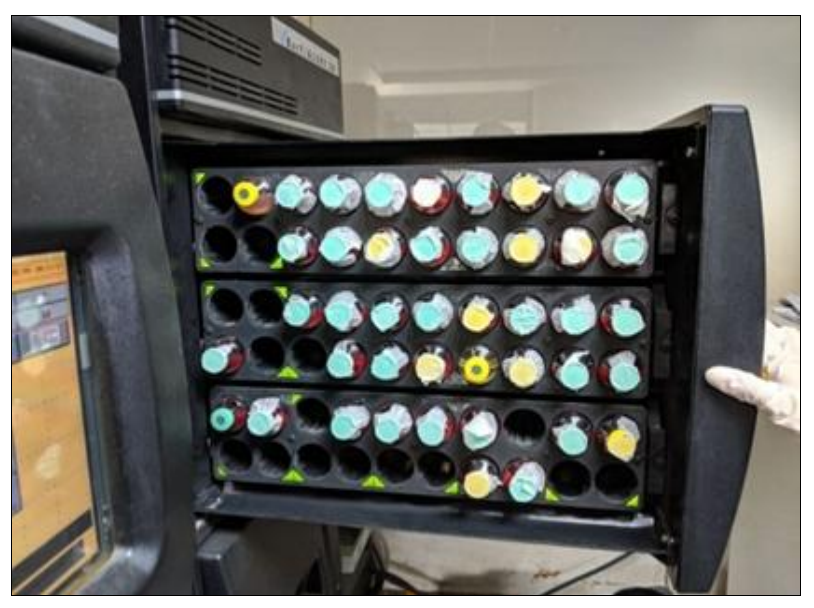

Fig 3: Loading Aerobic \& Anaerobic Culture Bottle In Bact/Alert

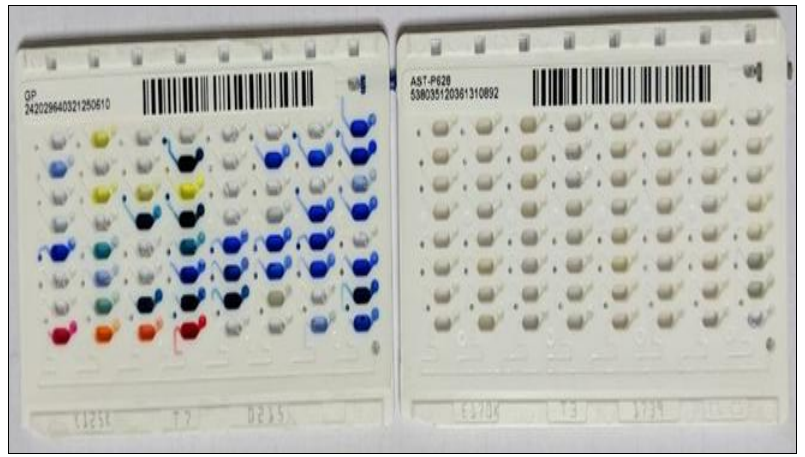

Fig 4: Bacterial \& Ast Culture Template Of Gram Positive Bacteria

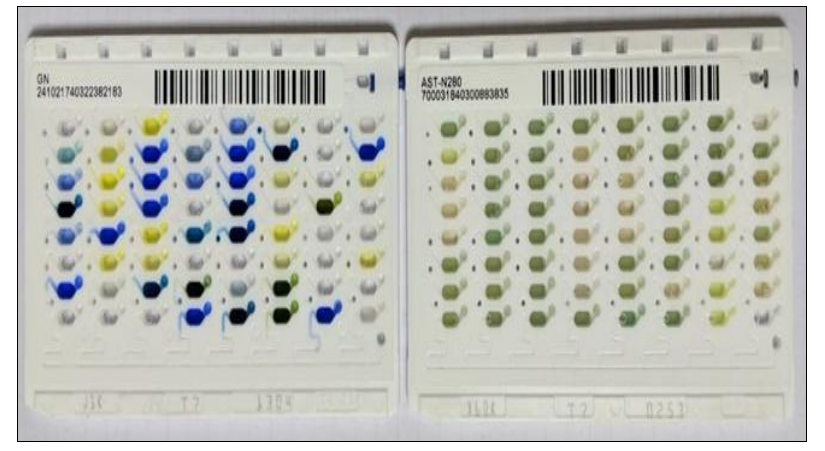

Fig 5: Bacterial \& Ast Culture Template of Gram Negative Bacteria

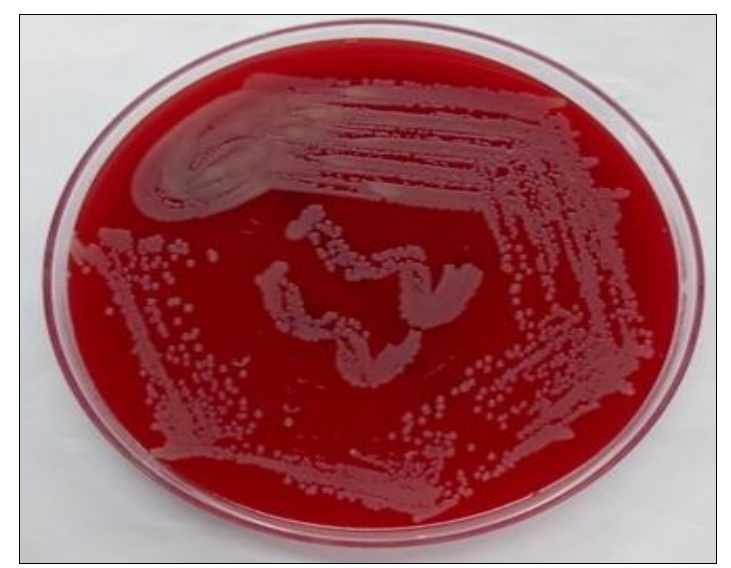

Fig 6: Culture Plate of E.Coli

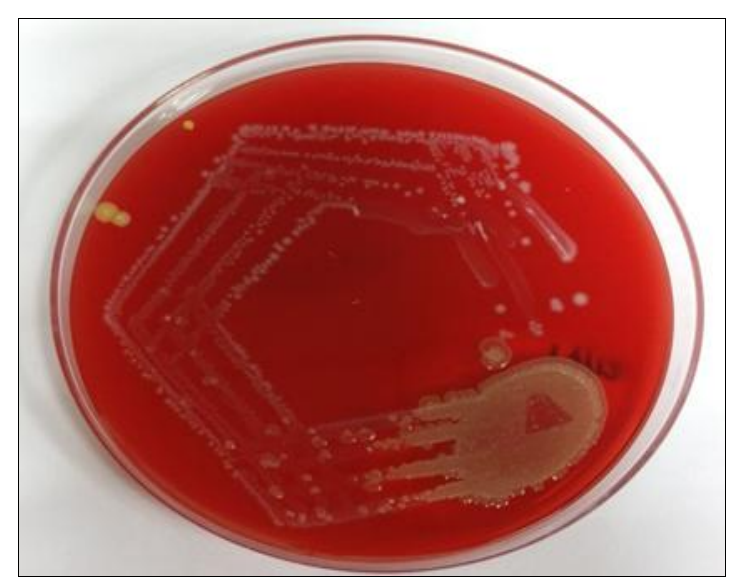

Fig 7: Culture Plate of E. Faecalis 


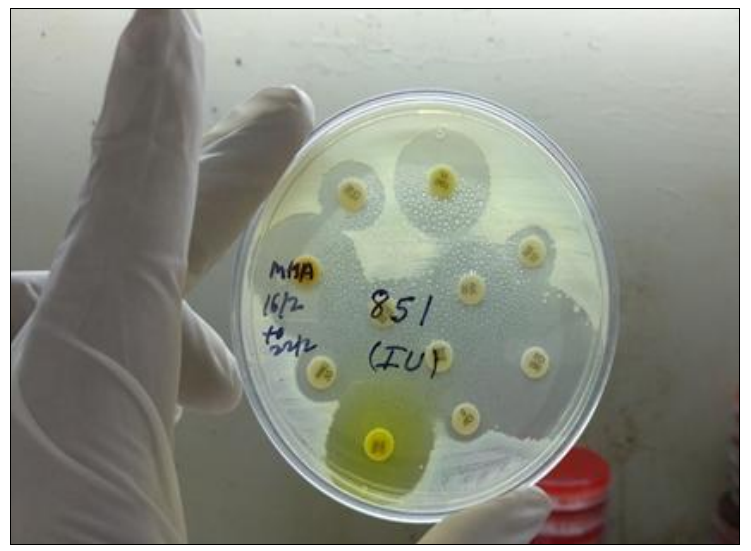

Fig 8: Antibiotic Susceptibility Testing of E.Coli

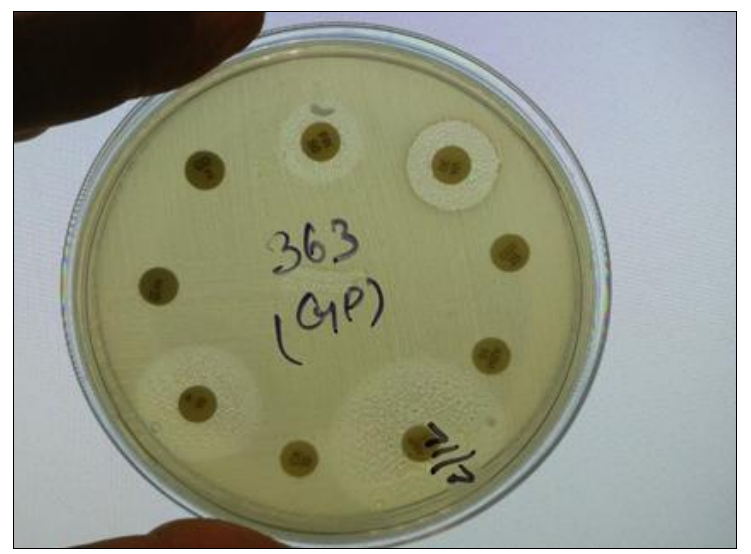

Fig 9: Antibiotic Susceptibility Testing Of E.Faecalis

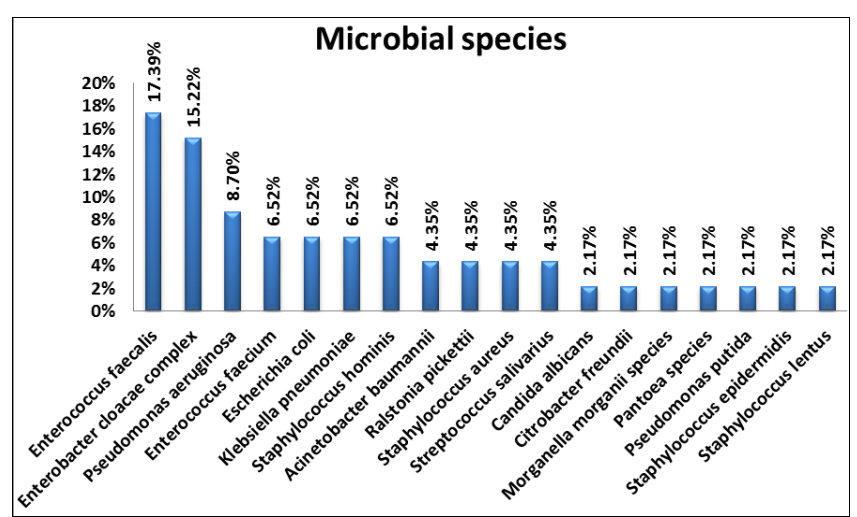

Graph 1: Microorganisms found in root canals of failed endodontic patients $(n=46)$

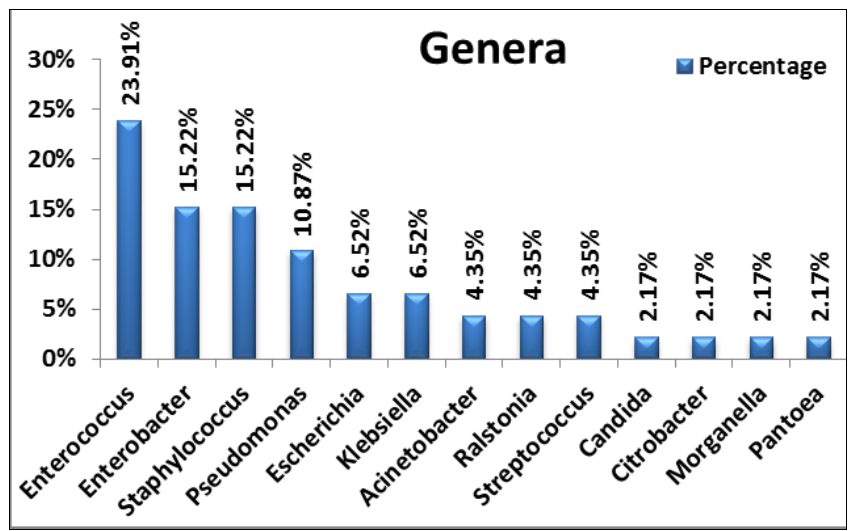

Graph 2: Prevalence of bacterial genera found

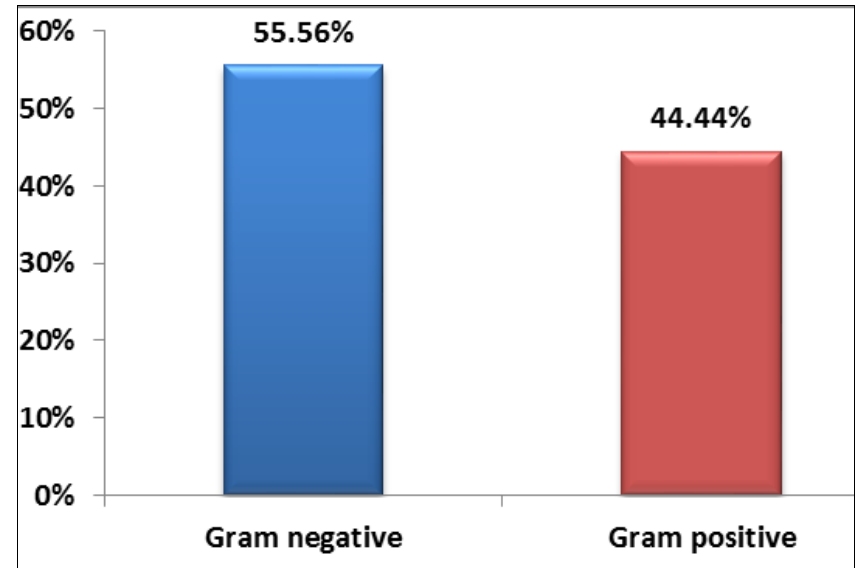

Graph 3: Prevalence of gram positive and gram negative bacteria

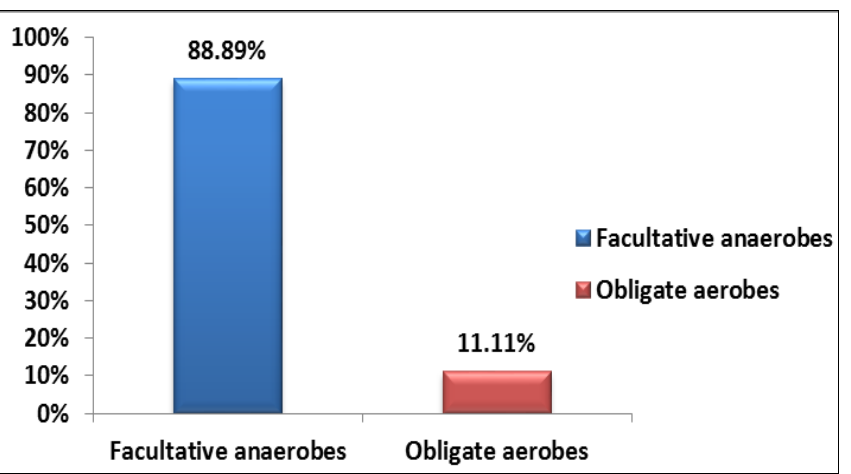

Graph 4: Prevalence of facultative anaerobes and obligate aerobes bacteria

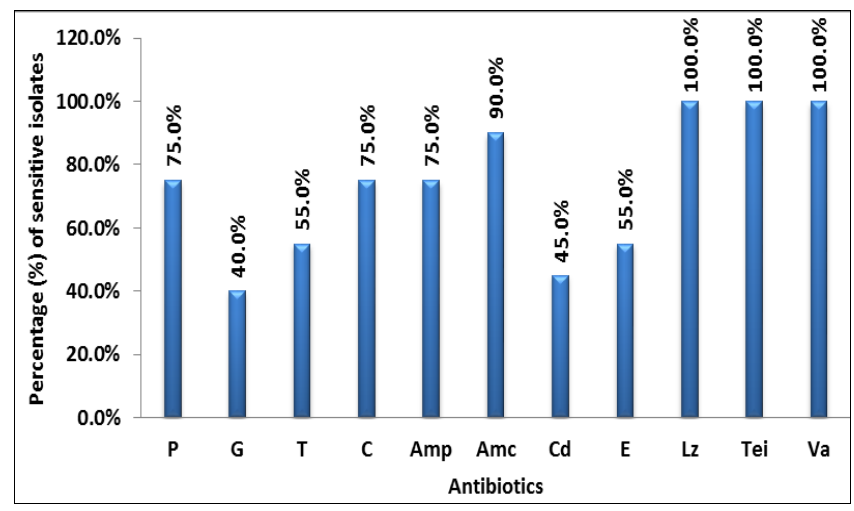

Graph 5: Antibiotic sensitivity pattern of Gram positive organisms

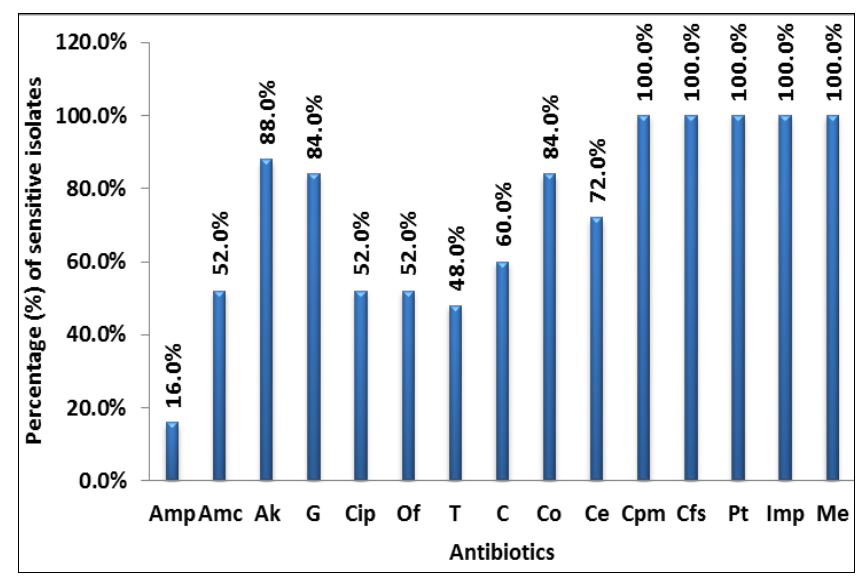

Graph 6: Antibiotic sensitivity pattern of Gram negative organisms. 


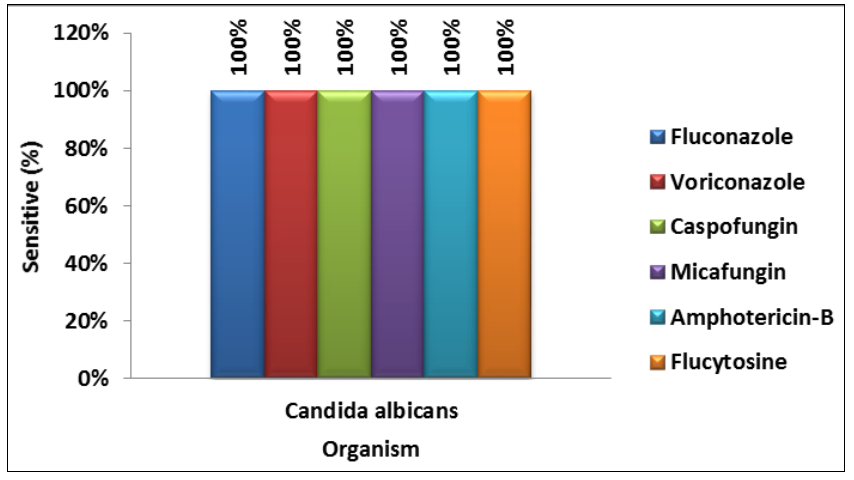

Graph 7: Antibiotic sensitivity pattern of Candida

\section{Conclusion}

The purpose of this study was to investigate and analyse the microbiological flora of root canals in endodontic failure cases and to find out the antibiotic susceptibility of these microorganisms. The fact that Enterococcus spp. was found in majority of cases indicates that Enterococcus is the predominant bacteria responsible for endodontic failure in a particular sub-population. Susceptibility and antibiotic sensitivity of different bacteria were analyzed and it was found that linezolid, teicoplanin \& vancomycin were the most effective antimicrobial against gram positive bacteria and Amikacin, Gentamycin, Cefotaxime, Cefepime, Cefoperazone, Piperacillin/tazobactam, Imipenem and Meropenam were the most effective antimicrobial against the gram negate e bacteria for root canal failure.

\section{References}

1. Fabricius L. Oral bacteria and apial periodontitis: an experimental study in monkeys [Thesis]. University of Goteborg (Sweden), 1982.

2. Alcoforado GAP, McKay TL, Slots J. Rapid method for detection of lactose fermentation oral microorganisms. Oral Microbiology and Immunology, 1987, 2.

3. Baumgartner JC, Falker WA. Bacteria inthe apical 5mmof infected root canals. Journal of Endodontics 1991;17:380^3.

4. Engstrom B. The significance of enterococci in root canal treatment. Odontologisk Revy 1964;15:87-106.

5. Fabricius L, Dahlen G, O hman AE, Mo ller AJR. Predominant indigenous oral bacteria isolated from infected root canals after varied time of closure. Scandinavian Journal of Dental Research 1982;90;13444.

6. Tronstad L, Barnett F, Riso K, Slots J. Extraradicular endodontic infections. Endod Dent Traumatol 1987;3:8690.

7. Evans M, Davies JK, Sundqvist G, Figdor D. Mechanisms involved in the resistance of Enterococcus faecalis to calcium hydroxide. Int. Endodontic $\mathbf{J}$ 2002;35:221-8.

8. Molander A, Reit C, Dahlen G, Kwist T. microbiological status of root filled teeth with apical periodontitis. Int. Endod J 1998:31 1-7.

9. Sundquist G, Fidgor D, Sjogren U. microbiology analyses of teeth with endodontic treatment and outcome of conservative retreatment. Oral Surg Oral Med Oral Pathol 1998:85:86-93.

10. Peculiene V, Balciuniene I, Eriksen HM, Haapasalo M. Isolation of Enterococcus faecalis in previously root filled teeth in a Lithuanian population. J Endod 2000:26:593-595.
11. Peculiene V, Reynaud AH, Balciuniene I, Haapasalo M. isolation of yeast and enteric bacteria in root filled teeth in chronic apical periodontitis. Int. Endod J 2001:34:429434.

12. Hung-Chih Chien, You Chan et al. Antibiotic susceptibility of microflora from unsuccessful endodontic treatment. J Dent Sci 2008;3(2).

13. Heena Sadiq, Priti Gupta et al. isolation, identification and sensitivity of the star microorganisms of the infected root canal: Enterococcus faecalis. Int. $\mathrm{J}$ of Scientific study $2015 ; 3(2)$. 